\title{
Brain activity
}

\section{Developing nanotech tools for neuroscience.}

At the start of 2013, the European Union awarded one of the two Future and Emerging Technology 'flagship' initiatives to the Human Brain Project (the other one going to a project focused on graphene ${ }^{1}$ ). Almost simultaneously, President Barack Obama announced the BRAIN (Brain Research through Advancing Innovative Neurotechnologies) Initiative in the US.

As one of the flagships initiatives, the Human Brain Project is due to receive a staggering $€ 1$ billion over 10 years, half of which will come from the European Union and other half from the funding agencies of the individual countries involved. It is a large collaboration involving over 100 partners, and $€ 72$ million ( $\sim$ US $\$ 98$ million) will be awarded during the first 30 months alone. The principal goals of the project are to simulate the activity of a human brain using supercomputers and to use the knowledge obtained to improve the way computers work.

The BRAIN initiative originated from a call from a large number of scientists to launch a collaborative effort, which was named the Brain Activity Map project, to record and analyse the activity of large sets of neurons in the brain. This call was answered by the White House who backed it with the promise of several hundred million dollars of public funding over the next few years and called for support from private investors. So far, about US\$110 million have been committed by the Defense Advanced Research Projects Agency, National Institutes of Health and National Science Foundation for the first year, and private investors have promised around US\$130 million for each of the next few years ${ }^{2}$.

The initiatives originate from two simple facts. First, that our current understanding of how the brain works is very poor, which hampers the discovery of effective cures for mental health diseases. Second, that the neuron network in the human brain is extremely vast and complex. Understanding the way in which signals are transmitted and how these transmissions translate into thoughts and sensations can only be achieved through large collaborations, which are able to produce and analyse huge sets of data. It is, therefore, no coincidence that the BRAIN initiative has been compared with endeavours such as the Human Genome Project and even the Apollo project that landed a man on the Moon.

Nanotechnology has a notable role to play in these initiatives. In particular, it can

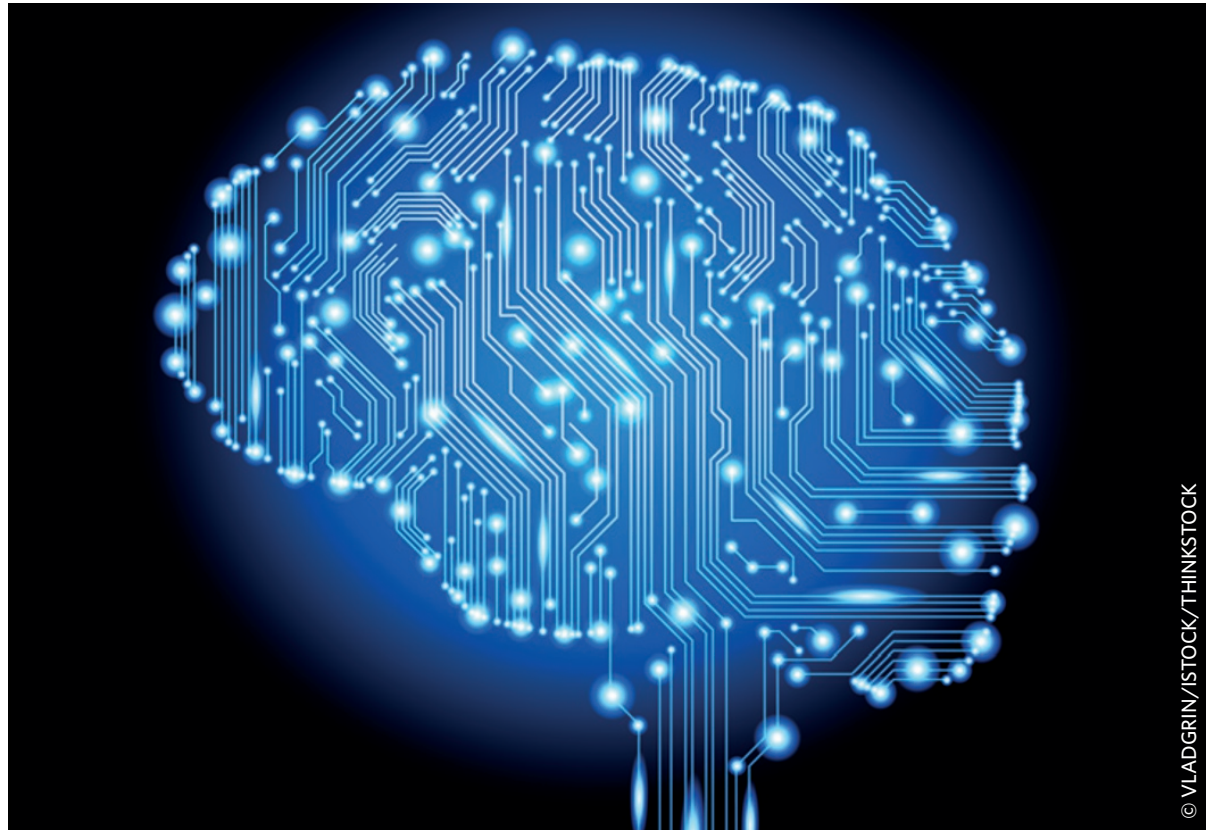

provide powerful tools for mapping the activity of neurons. The potential of such nanotechniques was extensively outlined in an article in ACS Nano last year ${ }^{3}$. The techniques suggested include the use of electrodes to measure intracellular and intercellular activity; optical techniques with improved lateral resolution that are able to detect signals from buried regions of the brain; the use of nanoparticles to monitor and report the chemical activity of labelled cells; and the fabrication, through synthetic biology, of hybrid systems that can facilitate optical or electrical signal detection from the brain.

A line of research that has been of particular interest recently is the development of nanoscale electrodes for mapping neuron activity ${ }^{4,5}$. Microelectrode arrays have traditionally been used for such purposes, but they cannot record intracellular signals and their spatial resolution is limited. Arrays of nanoscale electrodes, on the other hand, can detect intracellular signals and even stimulate individual neurons ${ }^{4}$. On page 142 of this issue of Nature Nanotechnology, Charles Lieber and colleagues report the development of a device based on a single nanowire that can be manipulated in three dimensions and used to probe the intracellular signals of targeted cells ${ }^{6}$. Although the device has so far only been tested on cardiac muscle cells, its extension to neurons should be feasible.
There are also serious challenges with such nanotechnologies for neuroscience. The main one is the size of the problem. There is an estimated $10^{10}$ to $10^{11}$ neurons in the human brain, each of them interacting with thousands of neurons around them. Although it is possible to imagine scaling up nanotechniques to record the activity of several thousand neurons, that would still be several orders of magnitude short of the final goal. Another challenge is to study the brain in realistic conditions. Techniques using nanoelectrodes, for example, can easily be applied to neuron circuits in culture, but these cultures are far from representing living tissue.

These problems can only be solved by starting from the basics and proceeding step by step. A full understanding of even a small number of neurons is still missing. In the meantime it may become more obvious how to extrapolate results on small number of neurons to larger circuits. It is unlikely that either initiative will provide a full understanding of the human brain in the next ten years. But we can expect significant steps forward and nanotechnology can play an important part in achieving them.

References

1. Nature Nanotech. 8, 221 (2013).

2. Nature 503, 26-28 (2013).

3. Alivisatos, P. A. et al. ACS Nano 7, 1850-1866 (2013).

4. Robinson, J. T. et al. Nature Nanotech. 7, 185-190 (2012).

Spira, M. E. \& Hai, A. Nature Nanotech. 8, 83-94 (2013).

Qing, Q. Nature Nanotech. 9, 142-147 (2014). 